\section{Púrpura trombopénica idiopática e hipotiroidismo primario en una paciente de 49 años}

\section{Sr. Director:}

La púrpura trombopénica idiopática (PTI) o enfermedad de Werlhof es una trombocitopenia periférica inmunológica crónica que se diagnostica por exclusión como una trombocitopenia aislada con megacariocitos aumentados en médula ósea, en ausencia de enfermedades subyacentes, y no atribuible a otras causas (infecciones, tóxicos o medicamentos). El diagnóstico diferencial debe realizarse con otros procesos que pueden cursar con púrpura como infecciones por VIH, coagulopatías, neoplasias, amiloidosis, colagenosis, síndrome de Marfan, enfermedad de Whipple y otros. Se relaciona con la presencia de anticuerpos antiplaqueta. Cursa en forma de brotes hemorrágicos de predominio cutáneo y el tratamiento se basa en la administración de glucocorticoides a dosis altas, esplenectomía como segunda opción y tratamiento con inmunosupresión si persiste la trombopenia sintomática ${ }^{1}$.

El hipotiroidismo primario es el síndrome clínico y bioquímico resultante de la disminución en la producción hormonal de la glándula tiroides, lo que conlleva un déficit de todos los procesos metabólicos que afectan a la fisiología de la mayoría de los sistemas y órganos. La prevalencia del hipotiroidismo clínico es de 1-2 /1.000 habitantes (de 5-20/1.000 entre los individuos que solicitan servicios médicos) ${ }^{2}$, ocupando la tiroiditis crónica autoinmune el primer lugar etiológico en las áreas con aporte de yodo adecuado. La relación varones/mujeres es de 1/2-8 y la incidencia aumenta con la edad ${ }^{2}$.

La coexistencia de estas dos patologías es poco frecuente como se observa a la luz de la literatura revisada ${ }^{3-5}$. En algunos casos dicha coexistencia está incluida en el seno de múltiples patologías: diabetes mellitus, miastenia gravis, lupus eritematoso sistémico, carcinoma basocelular, etc $^{3}$. La implicación inmunológica en ambos procesos puede sugerir una posible relación entre ellos, así como con otras entidades que cursan con alteración de la inmunidad.

\section{CASO CLÍNICO}

Presentamos el caso de una paciente de 49 años sin antecedentes familiares de interés. Como antecedente personal destaca una PTI diagnosticada en 1987 que precisó esplenectomía dos años más tarde; histerectomía más doble anexectomía por metrorragias en 1996, practicándose apendicetomía en el mismo acto quirúrgico, glaucoma, hipertensión, obesidad (índice de masa corporal $=32,7$ ) y síndrome del túnel carpiano bilateral.

Es remitida al Servicio de Endocrinología desde el Servicio de Reumatología donde estaba siendo revisada por un síndrome del túnel carpiano bilateral, al presentar las siguientes hormonas tiroideas: triyodotironina (T3) $70 \mathrm{ng} / \mathrm{dl}$ 
(60-80 ng/dl), tiroxina total (T4) 4 $\mu \mathrm{g} / \mathrm{dl}(5-12 \mu \mathrm{g} / \mathrm{dl})$, T4 libre 0,4 $\mathrm{ng} / \mathrm{dl}(0,7-1,8 \mathrm{ng} / \mathrm{dl})$ y tirotropina $\begin{array}{lll}\text { (TSH) } & 220 & \mu \mathrm{UI} / \mathrm{ml} \quad(0,5-5\end{array}$ $\mu \mathrm{UI} / \mathrm{ml})$, con anticuerpos antimicrosomales de $557 \mathrm{UI} / \mathrm{ml}$ (<100 $\mathrm{UI} / \mathrm{ml}$ ) y anticuerpos antitiroglobulina $>944 \mathrm{UI} / \mathrm{ml}(<60 \mathrm{UI} / \mathrm{ml})$. Clínicamente refería decaimiento y cansancio de aproximadamente dos años de evolución, soportando muy mal el calor, ritmo intestinal normal, frecuentes jaquecas y refería torpeza mental.

La exploración mostraba un peso de $82,5 \mathrm{~kg}$, talla $159,5 \mathrm{~cm}$, tensión arterial 145/80 $\mathrm{mmHg}$. Bocio grado I . Resto sin alteraciones de interés.

Como resultados del estudio analítico: colesterol total 217 mg/dl (150-200 mg/dl), triglicéridos $143 \mathrm{mg} / \mathrm{dl}$ (50-170 mg/dl), HDL colesterol (lipoproteínas de alta densidad) $31 \mathrm{mg} / \mathrm{dl}$ (45-65 $\mathrm{mg} / \mathrm{dl}$ ), LDL colesterol (Lipoproteínas de baja densidad) 160 $\mathrm{mg} / \mathrm{dl}$ (100-130 mg/dl), GGT (gammaglutamiltransferasa) 40 UI/L (3-52 UI/L). Glucosa, creatinina, ácido úrico, calcio, fósforo, magnesio, hierro, GPT (transaminasa glutamicopirúvico), fosfatasa alcalina, iones y proteínas totales, dentro de la normalidad. En el hemograma aparece: leucocitos $8.000 / \mathrm{mm}^{3}$, con $6,46 \%$ de eosinófilos, $1,93 \%$ de basófilos, resto de la serie blanca normal. Hemoglobina $14,9 \mathrm{~g} / \mathrm{dl}$, hematocrito $43,8 \%$. Volumen corpuscular medio 89,6 fl. Plaquetas $33.000 / \mathrm{mm}^{3}$, VSG $5 \mathrm{~mm}$. Hormonas tiroideas: T3 120 ng/dl, T4 9,9 $\mu \mathrm{g} / \mathrm{dl}$, T4 libre $1,2 \mathrm{ng} / \mathrm{dl}$ y TSH $0,45 \mu \mathrm{UI} / \mathrm{ml}$. Anticuerpos antitiroideos: Ac-antimicrosomales $1.882 \mathrm{UI} / \mathrm{ml}$ (positivo superior a 100). Ac-antitiroglobulina $24.743 \mathrm{UI} / \mathrm{ml}$ (positivo superior a 100).

Radiografía lateral de cráneo: silla turca normal.

Electrocardiograma: sin alteraciones de conducción, voltaje ni repolarización.

El diagnóstico fue de hipotiroidismo primario por tiroiditis crónica.
La evolución de la paciente fue favorable tras iniciar tratamiento con $\mathrm{T} 4$ a dosis progresivas, encontrándose a los 9 meses clínica y hormonalmente eutiroidea. Al presentar un potasio de $3,7 \mathrm{mEq} / \mathrm{L}$, se hizo despistaje de hiperaldosteronismo primario, descartándose esta patología.

\section{DISCUSIÓN}

La clínica de la PTI es común a la de otras trombocitopenias (fragilidad capilar, tiempo de hemorragia prolongado y retracción deficiente del coágulo). Cursa fundamentalmente en forma de brotes hemorrágicos cutáneos, que se siguen de periodos de remisión de mayor o menor duración según la gravedad de la enfermedad. La morfología revela anisocoria plaquetaria (megatrombocitos junto con microtrombocitos). El diagnóstico se basa en la demostración de valores aumentados de PAIgG (inmunoglobulina $G$ asociada a plaquetas), siendo necesario el uso de pruebas cuantitativas porque en plaquetas normales se dan valores relativamente elevados de $\mathrm{IgG}^{6}$.

La etiología del hipotiroidismo primario comprende: déficit de yodo, tiroiditis crónica autoinmune, hipotiroidismo transitorio autoinmune, tratamiento con radioyodo, hipotiroidismo posquirúrgico, radioterapia externa, enfermedades infiltrativas, disgenesia tiroidea, defectos congénitos de la hormonogénesis tiroidea, agentes antitiroideos y exceso de yodo. La clínica es muy variable dependiendo de la rapidez de instauración, duración y gravedad, y deriva del enlentecimiento de las funciones orgánicas mediadas por las hormonas tiroideas. En el caso que nos ocupa la clínica se instauró lentamente, como suele ocurrir por lo general en los casos de tiroiditis autoinmune ${ }^{7}$.

Aunque la coexistencia de PTI con hipertiroidismo viene descrita con cierta frecuencia en la li- teratura médica ${ }^{8-10}$, no ocurre lo mismo con el hipotiroidismo y PTI, ya que encontramos referencias muy escasas y se remontan a los años 1970, 79 y $98^{3-5}$. Por esto y a pesar de contar con escasos antecedentes publicados, ante la posible relación entre estas entidades, no cabría descartar el estudio de autoanticuerpos en pacientes diagnosticados de PTI, así como en otros pacientes afectados por anemia hemolítica autoinmune y síndrome de Fisher- Evans, debido a la posibilidad de desarrollar hipotiroidismo a medio o largo plazo. Aunque quizás esta afirmación resulte un tanto aventurada en este caso debido al tiempo transcurrido entre el diagnóstico de PTI y el de hipotiroidismo (5 años). Investigaciones llevadas a cabo en Italia parecen confirmar un alto porcentaje de pacientes diagnosticados de PTI con autoanticuerpos antitiroideos elevados ${ }^{5}$, lo cual se corresponde con el caso aquí presentado.

\section{Galindo Dobón, C. A. Serrano Gállego, V. Estopiñán García ${ }^{1}$}

MIR $3^{\circ}$ Medicina Familiar y Co munitaria. Centro de Salud de Teruel. ' Unidad de Endocrinolo gía. Hospital Obispo Polanco. Teruel.

\section{BIBLIOGRAFÍA}

1. George JN, Woolf SH, Raskob GE, Wasser JS, Aledort LM, Ballem PJ, et al. Idiopathic thrombocytopenic purpura: a practice guideline developed by explicit methods for the American Society of Hematology. Blood 1996; 88: 3-40.

2. Utiger RD. Hypothyroidism. En: Felig P, Baxter JD, Frohman LA. Endocrinology and metabolism. $3^{\mathrm{a}}$ ed. Nueva York: McGraw Hill, 1995. p. 492-507.

3. Kornfeld P, Glass J, Papatestas AE, Horowitz SH, Matta R, Genkins G. Case report: Thymectomy-induced remission of acquired autoimmunes haemolytic anemia in an adult with myasthenia gravis. Am J Med Sci 1979; 277 (1): 111-6. 
4. Volpe JA, Johnston GS. Coexistent hypothyroidism and idiopathic thrombocytopenic purpura. Mil Med 1970; 135 (12): 1146-8

5. Lio S, Albin M, Girelli G, Perrone MP, Gandolfo G, Conti L, et al. Abnormal thyroid function test results in patients with Fisher- Evans syndrom. J Endocrinol Invest 1993; 16 (3): 163-7.

6. González R, Lozano ML, Moraleda JM. Diagnóstico de los trastornos de la hemostasia. En: González R, Lozano ML, Moraleda JM. Hematología. Madrid: Luzán, 1996. p. 459-69.

7. Dayan CM, Daniels GH. Chronic autoimmune thyroiditis. N Engl J Med 1996; 335: 99-107.

8. Hofbauer LC, Spitzweg C, Schmauss $\mathrm{S}$, Heufelder AE. Graves disease associated with autoimmune thrombocytopenic purpura. Arch Intern Med 1997; 157 (9): 1033-6.

9. Salgado V, Nieto Gámiz I, Fernández Sepúlveda S, Grilo Reina A. Púrpura trombocitopenica idiopática y enfermedad de Graves: Interrogantes patogénicos de una rara asociación. An Med Interna 1998; 15 (9): 509-10.

10. Aggarwal A, Doolittle G. Autoimmune thrombocytopenic purpura associated with hyperthyroidism in a single individual. South Med J 1997; 90 (9): 933-6.

\section{Síndrome de las piernas inquietas como causa de insomnio}

\section{Sr. Director:}

Presentamos el caso de una mujer de 62 años de edad, con antecedentes familiares de cáncer de pulmón e insomnio no filiado en el padre. Madre con antecedentes de hipertensión arterial. Como antecedentes personales presenta infecciones del tracto urinario de repetición no complicadas y dermatofitosis resuelta. Ha consultado en varias ocasiones por pirosis postpandrial y cefalea tensional. Amigdalectomizada e histerectomía por leiomioma a los 50 años.

Desde hace aproximadamente un año, viene refiriendo dolor, parestesias y disestesias en extre- midades inferiores de predominio distal. Estas alteraciones suelen comenzar por la tarde y se suelen prolongar toda la noche, hecho que le impide conciliar el sueño. También se asocia una necesidad imperiosa de mover las piernas y en ocasiones sacudidas nocturnas que la despiertan y que no le permiten tener un sueño reparador. Motivo éste por el que acude varias veces a la consulta de su médico de familia. En principio la paciente fue diagnosticada de insomnio debido a un cuadro ansioso-depresivo y se inició tratamiento con sertralina, sin cambios en la clínica. Por lo que se sospechó un síndrome de las piernas inquietas (SPI).

La exploración física fue normal, sistemático y bioquímica de sangre normales, pudiendo de esta manera descartar el origen secundario del SPI, así como de otros trastornos del movimiento, calambres nocturnos de las piernas, neuropatías periféricas y patología vascular de miembros inferiores. Se prescribió clonazepam a dosis de $0,5 \mathrm{mg}$ cada 12 horas, presentando tras una semana de tratamiento una mejoría completa, de forma que los síntomas han desaparecido; refiere dormir sin problemas durante 7 u 8 horas, desapareciendo las disestesias y la necesidad imperiosa de mover las piernas.

El SPI es un trastorno crónico del sistema central que con frecuencia origina un insomnio severo que puede ser incapacitante. Actualmente se cree que afecta hasta el $15 \%$ de la población, especialmente a la anciana ${ }^{1}$. Existe una alta incidencia de casos familiares, lo que sugiere un origen genético ${ }^{2}$, aunque también puede ser secundario, asociado a neuropatías periféricas, insuficiencia renal crónica (hasta el $40 \%$ de los pacientes en diálisis tienen el síndrome), anemia ferropénica, embarazo, incluso por drogas como antidepresivos tricíclicos, IRS ${ }^{3}$, litio y antagonistas de dopamina. Así como di- versas afecciones neurológicas (patología de médula espinal, radiculopatía lumbosacra, etc.). No hay evidencias de que el síndrome se relacione con trastornos psiquiátricos.

Para hacer el diagnóstico se requiere la presencia de cuatro elementos básicos: deseo de mover las piernas, generalmente asociado a parestesias y disestesias; la exacerbación de los síntomas con el descanso y el alivio con la actividad; inquietud motora y empeoramiento nocturno de los síntomas ${ }^{1}$. Criterios que cumple el caso clínico antes expuesto; ademas de fenómenos asociados como trastornos del sueño y fatiga diurna, examen neurológico normal y movimientos involuntarios de las piernas.

El diagnóstico de SPI se podrá hacer basándose únicamente en la historia, no estando indicada la polisomnografía. La American of Sleep Medicine publicó recientemente ${ }^{4}$ los parámetros prácticos para el tratamiento del síndrome. La selección de los fármacos estará influenciada por numerosos factores como son: la edad del paciente, la severidad de los síntomas, la frecuencia o regularidad de los síntomas, presencia de embarazo, insuficiencia renal, etc. Los fármacos dopaminérgicos son los mejor estudiados y más eficaces. Aunque la levodopa con carbidopa habitualmente se ha considerado la piedra angular del tratamiento, los agonistas de la dopamina (promipexol, pergolida) están ganando popularidad debido a que potencialmente son más eficaces y tienen menos fectos secundarios. Estudios recientes han mostrado que otro agente dopaminérgico, ropinirol ${ }^{5}$, y el nuevo anticonvulsivo, gabapentina, también son eficaces para controlar este síndrome ${ }^{6}$. Otros agentes de demostrada eficacia son las benzodiazepinas (clonazepam), opioides (codeína, oxicodona, propoxifeno) y la clonidina; fármacos estos de mayor manejo en Atención Primaria. 
El médico de familia juega un papel central a la hora de identificar e instaurar tratamiento adecuado en el síndrome de las piernas inquietas.

\section{J. P. Justel Pérez, A. Molina, J.C. Estévez, I. Mansilla}

Especialistas en Medicina Familiar y Comunitaria. Centro de Salud General Fanjul. Área 7. Madrid

\section{BIBLIOGRAFÍA}

1. Walters AS. Toward a better definition of the restless legs syndrome. Mov Disord 1995; 10: 634-42.

2. Strang RR. The symptom of restless legs. Med J Aust 1967; 1: 1211-3.

3. Bakshi R. Fluoxetine and restless legs syndrome. J Neurol SCI 1996; 142: $151-2$.

4. Chesson Al Jr, Wise M, Dávila D, Sindro S, Littner $M$, Anderson WM, et al. Syndrome and periodic limb movement disorder. An Ame- rican Academy of Sleep Medicine Report. Standards of Practice Committee of the American Academy of Sleep Medicine. Sleep 1999; 22. 961-8.

5. Estevill-Sancho EX, De la FuentePanell V. Ropirinol as a succesful treatment of the restless legs syndrome: an open pilot study with polisomnographic data. Sleep 1999; 22 (Supl. 1): 158.

6. Uhles ML, Duntley SP. Evaluation of the efficacy of neurontin in the treatment of restless legs syndrome sleep 1999; 22 (Supl. 1): 157S.

\section{FE DE ERRATAS}

En el artículo editorial del volumen 12, número 8 de la revista MEDIFAM, hubo un error en la tabla IV "Algunos datos del PMASS" al final de la primera columna, por lo que a continuación reproducimos la tabla con los datos correctos.

\begin{tabular}{|c|c|c|c|c|}
\hline \multicolumn{5}{|c|}{ ALGUNOS DATOS DEL PMASS } \\
\hline & 1998 & 1999 & 2000 & 2001 \\
\hline $\begin{array}{l}\text { Procedimiento para concertar cita } \\
\text { Teléfono ( } \% \text { usuarios) } \\
\text { Mostrador ( } \% \text { usuarios) }\end{array}$ & $\begin{array}{l}67,7 \\
32,3\end{array}$ & $\begin{array}{l}67,4 \\
32,6\end{array}$ & $\begin{array}{l}67,3 \\
32,7\end{array}$ & $\begin{array}{l}65,4 \\
34,6\end{array}$ \\
\hline Relación horario apertura/ horario cita & 0,9 & 0,9 & 0,9 & 0,9 \\
\hline $\begin{array}{l}\text { Acumulación de llamadas tramos } \\
\text { horarios (\% centros) } \\
\text { De } 8 \text { a } 11 \text { horas } \\
\text { De } 15 \text { a } 18 \text { horas }\end{array}$ & $\begin{array}{l}67,1 \\
10,0\end{array}$ & $\begin{array}{c}70,7 \\
9,0\end{array}$ & $\begin{array}{l}67,5 \\
13,9\end{array}$ & $\begin{array}{l}68,9 \\
13,1\end{array}$ \\
\hline $\begin{array}{l}\text { Llamamiento inadecuado (\% consultas) } \\
\text { Paciente saliente } \\
\text { Exposición lista } \\
\text { Bloques } 1 / 4 \text { hora }\end{array}$ & $\begin{array}{c}9,4 \\
12,4 \\
15,2\end{array}$ & $\begin{array}{c}8,5 \\
12,6 \\
15,2\end{array}$ & $\begin{array}{c}8,2 \\
12,4 \\
13,1\end{array}$ & $\begin{array}{c}8,0 \\
12,2 \\
13,1\end{array}$ \\
\hline $\begin{array}{l}\text { Citación con A. Especializada ( } \% \text { citas) } \\
\text { A tiempo real } \\
\text { A tiempo diferido }\end{array}$ & $\begin{array}{l}26,5 \\
73,5\end{array}$ & $\begin{array}{l}29,4 \\
70,3\end{array}$ & $\begin{array}{l}34,3 \\
65,7\end{array}$ & $\begin{array}{l}39,5 \\
60,5\end{array}$ \\
\hline $\begin{array}{l}\text { Promedio días tramitación Visado Rec, } \\
\text { № total de reclamaciones } \\
\text { \% Reclamaciones estructura } \\
\text { \% Reclamaciones proceso } \\
\% \text { Recl. contestadas }<30 \text { días } \\
\% \text { Recl. que han requerido actuación }\end{array}$ & $\begin{array}{c}4,1 \\
19.340 \\
12,0 \\
84,5 \\
79,6 \\
17,0\end{array}$ & $\begin{array}{c}3,9 \\
20.163 \\
13,8 \\
82,2 \\
77,5 \\
16,1\end{array}$ & $\begin{array}{c}3,6 \\
21.051 \\
12,8 \\
80,3 \\
83,0 \\
11,5\end{array}$ & $\begin{array}{c}3,1 \\
23.816 \\
13,5 \\
82,9 \\
79,4 \\
18,4\end{array}$ \\
\hline
\end{tabular}

En la última línea se hacía referencia a "\% Recl. que han requerido contestación", debiendo figurar "\% Recl. que han requerido actuación". 